\title{
Impact of safety factor and magnetic shear profiles on edge turbulence in circular limited geometry
}

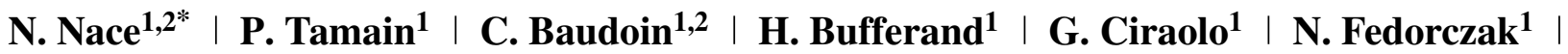 \\ D. Galassi ${ }^{2} \mid$ Ph. Ghendrih ${ }^{1} \mid$ E. Serre ${ }^{2}$
}

${ }^{1}$ IRFM, CEA Cadarache, France

${ }^{2}$ Aix Marseille Univ, CNRS, Centrale Marseille, M2P2, Marseille, France

*Correspondence

N. Nace, IRFM, CEA Cadarache, F-13108

St-Paul-lez-Durance, France.

Email: nace.nicolas@gmail.com

Funding Information

This research was supported by the CINECA. TGCC, A0010506912. GENCI IDRIS,

x2016056912. Equip@ Meso,

ANR-10-EQPX-29-01. French Government.

A*MIDEX, ANR-11-IDEX-0001-02. Agence

Nationale pour la Recherche (ANR).
The impact of magnetic configuration on edge turbulence properties in circular limiter geometry is investigated using TOKAM3X, a three-dimensional (3D), first-principle, fluid code for edge plasma. The theoretical spatial tilting of magnetic shear on turbulence fluctuations is recovered. Magnetic shear is found to generate or enhance poloidal high/low field sides (HFS/LFS) and up/down asymmetries. A simulation mimicking the impact of an X-point on circular limiter geometry leads to the formation of two transport barriers that are stable in time, thus leading to the improvement of core particle confinement and to reduction of radial turbulent transport. The magnetic shear, which also strongly enhances the $E \times B$ shear, is responsible for the barrier formation.

\section{KEYWORDS}

edge, magnetic shear, simulations, tokamak, transport barrier, turbulence

\section{1 | INTRODUCTION}

The H-mode power threshold is found to be reduced in divertor compared to limiter tokamaks. ${ }^{[1]}$ One of the differences between these two configurations is the presence of an X-point in the divertor case characterized by a zero magnetic field pitch angle, which locally generates a strong magnetic shear. Other key quantities related to the edge plasma are linked to the poloidal magnetic field amplitude, such as the power scrape-off layer (SOL) width scaling law or the Greenwald limit (through plasma current), but the role of the pitch angle and magnetic shear on these laws has not been established. The magnetic shear is also able to impact turbulence by generating a spatial tilting of fluctuations, which modifies the poloidal asymmetries of edge plasma. ${ }^{[2]}$ The present study aims to evaluate the role played by safety factor and magnetic shear profiles on edge transport properties and to investigate the existence of spontaneous edge transport barriers generated by this magnetic shear. For this purpose, the fluid turbulence code TOKAM3X is used. It solves three-dimensional (3D) fluid drift equations in edge plasma in a global and flexible magnetic geometry, ${ }^{[3]}$ thus providing the freedom to investigate the impact of magnetic configuration. Both the magnetic configuration and the TOKAM $3 \mathrm{X}$ code are detailed in section 2 . The effect of safety factor and magnetic shear on edge transport properties is then analysed in section 3, especially in terms of the pattern of density structures and poloidal asymmetries. Finally, section 4 focuses on the analysis of an edge transport barrier obtained using a magnetic configuration mimicking the radial profile of cylindrical safety factor and magnetic shear in an X-point configuration.

\section{2 | THREE-DIMENSIONAL EDGE TURBULENCE SIMULATIONS IN CIRCULAR LIMITED GEOMETRY}

TOKAM3X is a 3D flux-driven turbulence code for edge plasma-including both open and closed field lines in the vicinity of the last closed magnetic surface (LCFS) — based on drift-reduced Braginskii equations. The code is used in its 
isothermal version with constant and uniform ion and electron temperatures $T_{i}=T_{e}=1$. Consequently, the equations solved by TOKAM3X are the particle balance, ${ }^{[1]}$ the ion parallel momentum balance, ${ }^{[2]}$ the vorticity balance, ${ }^{[4]}$ the parallel Ohm's law, ${ }^{[3]}$ and the expression of the so-called generalized vorticity, ${ }^{[5]}$ which takes into account diamagnetic effects in the ion polarization drift:

$$
\begin{gathered}
\partial_{t} N+\nabla \cdot\left(N\left(u_{i_{\|}} \mathbf{b}+\mathbf{u}_{\perp}^{E \times B}\right)\right)=\nabla \cdot\left(D_{N} \nabla_{\perp} N\right)+S_{N} \\
\partial_{t} \Gamma_{i_{\|}}+\nabla \cdot\left(\Gamma_{i_{\|}}\left(u_{i_{\|}} \mathbf{b}+\mathbf{u}_{\perp}^{E \times B}\right)\right)=-2 \nabla_{\|} N+\nabla \cdot\left(D_{\Gamma_{i}} \nabla_{\perp} \Gamma_{i_{\|}}\right) \\
\partial_{t} W+\nabla \cdot\left(W\left(u_{i_{\|}} \mathbf{b}+\mathbf{u}_{\perp}^{E \times B}+\mathbf{u}_{i_{\perp}}^{\nabla B}\right)\right)=\nabla \cdot\left(N\left(\mathbf{u}_{i_{\perp}}^{\nabla B}-\mathbf{u}_{e_{\perp}}^{\nabla B}\right)\right)+\nabla \cdot\left(j_{\|} \mathbf{b}\right) \\
+\nabla \cdot\left(D_{W} \nabla_{\perp} W\right) \\
\eta_{\|} N j_{\|}=\nabla_{\|} N-N \nabla_{\|} \varphi \\
W=\nabla \cdot\left(\frac{1}{B^{2}} \nabla_{\perp} \varphi+\frac{1}{N B^{2}} \nabla_{\perp} N\right)
\end{gathered}
$$

In these normalized equations, time and length are, respectively, normalized to gyro-frequency $\omega_{c_{i}}=e B_{0} / m_{i}$ and gyro-radius $\rho_{L}=\sqrt{m_{i} T_{0}} / e B_{0}$, where $B_{0}$ and $T_{0}$ are, respectively, the reference magnetic field and the reference temperature. $N, \varphi$, $W$, and $j_{\|}$are, respectively, the normalized density, electrostatic potential, vorticity, and parallel current. $u_{i_{\|}}$is the ion parallel momentum, and $\Gamma_{i_{\|}}=N u_{i_{\|}}$is the associated ion parallel flux. The perpendicular transport is modelled through fluid drifts and diffusive processes. The formers are composed of the $\mathbf{E} \times \mathbf{B}$ and $\nabla B$ drifts defined in their normalized form as: $\mathbf{u}_{\perp}^{E \times B}=(\mathbf{b} \times \nabla \varphi) / B$ and $\mathbf{u}_{i / e_{\perp}}^{\nabla B}= \pm 2(\mathbf{b} \times \nabla B) / B^{2}$, while the ion polarization drift is used to obtain the vorticity balance Equation 3. Diffusive transport is ensured through constant diffusive coefficients $D_{N}, D_{\Gamma_{i}}$, and $D_{W}$, equal to $5 \times 10^{-3} \rho_{L} c_{s}$ in our simulations. $\eta_{\|}=10^{-5} B_{0} / e N_{0}$ is the normalized parallel collisional plasma resistivity with an amplitude about 10 times larger than the one expected for a device like Tore Supra to reduce numerical cost. The flux-driven approach is ensured by the volumetric particle source $S_{N}$, which drives the particle flux coming from core plasma such that no scale separation between equilibrium and fluctuations has to be assumed. Considering the geometrical aspect, we choose an aspect ratio equal to 3.75 and $\rho_{*}^{-1}=a / \rho_{L}=256$. More details on the normalization procedure, boundary conditions, and numerical aspects can be found in Tamain et al. ${ }^{[3]}$

In order to focus on the impact of pitch angle, we consider cases with the minimum geometrical complexity—no poloidally varying flux expansion and no X-point—and consequently opt for limited plasmas in circular geometry. The toroidal magnetic field - decreasing with major radius $R$-is kept identical in all the simulations presented in this study, and the poloidal magnetic field is adapted in order to obtain the expected magnetic configuration, especially in terms of safety factor and magnetic shear radial profiles. Our scanning parameter is thus the cylindrical safety factor $q^{\mathrm{cyl}}=\left\langle r B_{\mathrm{tor}} /\left(R B_{\mathrm{pol}}\right)\right\rangle_{\theta}$, where definition can be extended even in open field lines. From this definition, the magnetic shear is defined as $s^{\mathrm{cyl}}=\left\langle r / q^{\mathrm{cyl}} d q^{\mathrm{cyl}} / d r\right\rangle_{\theta}$. Three simulations are compared in this study, and the corresponding radial profiles of cylindrical safety factor and magnetic shear are presented, respectively, in Figures 1 and 2. In the next section, we first compare results obtained with the realistic $q^{\text {cyl }}$-profile for such circular limiter geometry—which has a parabolic shape increasing from 3 at the core edge $(r=0.8 a)$ to 6 at the far SOL $(r=1.2 a)$ - to a case without magnetic shear, that is, a flat $q^{\text {cyl }}$-profile. This constant safety factor is chosen to be equal to 3.85 , corresponding to the value of the parabolic profile at the LCFS. In section 4, a Gaussian shape centred around the LCFS is superimposed on the parabolic $q^{\text {cyl }}$-profile in order to mimic the effect of an X-point. To determine the amplitude of this Gaussian, we compute the flux surface average value of the magnetic shear obtained in a COMPASS-like divertor simulation including an X-point. One can observe in Figure 2 two strong peaks - a positive and a negative one-of magnetic shear on both sides of the LCFS. The safety factor and magnetic shear profiles and amplitudes obtained are consistent with those of the COMPASS-like divertor simulation. However, our simulation does not exhibit strong poloidal variation of cylindrical safety factor and magnetic shear, and we only focus on the mean impact of magnetic shear on transport properties rather than the local effect around the X-point as in the realistic divertor case. Note that this strong hypothesis can impact turbulence properties: for example, the magnetic shear is expected to have an impact on interchange and drift-wave instability growth rates. ${ }^{[4]}$ 

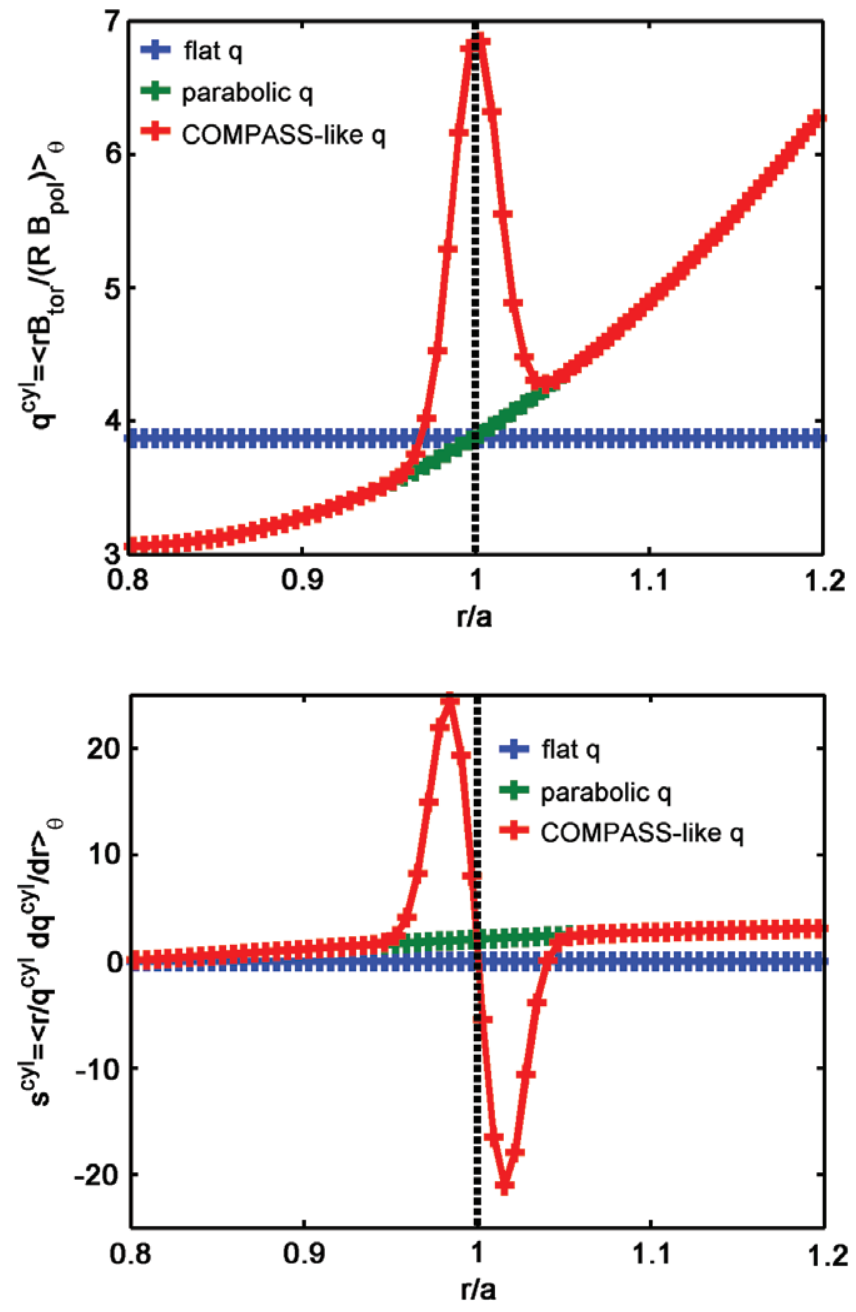

FIGURE 1 Radial profiles of cylindrical safety factor $q^{\text {cyl }}$

FIGURE 2 Radial profiles of cylindrical magnetic shear $s^{\mathrm{cyl}}$

\section{I IMPACT OF SAFETY FACTOR AND MAGNETIC SHEAR ON EDGE TRANSPORT PROPERTIES}

The theoretical effect of magnetic shear on turbulence is to generate a spatial tilting of ballooned fluctuations excited at outboard mid-plane. ${ }^{[2]}$ In particular, we expect a radial elongation of turbulent structures crossing a magnetic shear region associated with a reduction of the poloidal elongation, the effect being larger when the poloidal distance from outboard mid-plane increases. It results in an asymmetric pattern with respect to the low field side (LFS) mid-plane. In order to confirm that this effect of magnetic shear on turbulence is significant, a conditional averaging is realized on density fluctuations. The procedure is the following: first, the poloidal plane is split into 17 parcels; the closed field lines region is divided into 8 parcels with the same poloidal extension of $45^{\circ}$, while the SOL is split into 7 parcels of $45^{\circ}$ and 2 parcels of $22.5^{\circ}$ on both sides of the limiter. In each parcel, a conditional averaging on density is performed on the centre, and only rare fluctuating events exceeding 2 standard deviations are considered. For these events, we average the corresponding density fluctuations in the whole parcel. Density patterns of large events resulting from this conditional averaging procedure can be observed in Figure 3 for the simulation with a flat $q^{\text {cyl }}$-profile and in Figure 4 for the simulation with parabolic $q^{\text {cyl }}$-profile.

Without magnetic shear, one can observe in Figure 3 that the pattern of density fluctuations is quite circular and mostly isotropic on both open and closed field lines. No strong differences can be observed in the shape of fluctuations between high and low field sides, even if density structures seem to be slightly more elongated in the poloidal direction on the high field side (HFS). Conversely, the patterns of density fluctuations are strongly impacted when the magnetic shear is included (Figure 4). First, on the LFS mid-plane, density fluctuations reveal a more potato-like shape, and the size of the structures have strongly increased. One can notice a spatial tilting of turbulent structures in the poloidal direction: the expected asymmetry of density patterns ${ }^{[2]}$ with respect to the LFS mid-plane is recovered. When the poloidal distance with LFS mid-plane increase, the spatial tilting keeps amplifying, especially in the open field line region, which can be explained as the connection between LFS and HFS being weaker in the SOL in comparison to the closed field lines region, where a strong coupling exists between HFS and LFS. The magnetic shear is found to have a strong impact on density fluctuations, with a differential effect on low and high field sides. One may wonder if these differences of density fluctuation patterns have an impact on transport properties. To 
FIGURE 3 Conditional averaging on density fluctuations for flat $q^{\mathrm{cyl}}$-profile. The poloidal section is split into 17 parcels delimited by dashed black lines. The conditional averaging procedure is applied on the white circle on the centre of each parcel
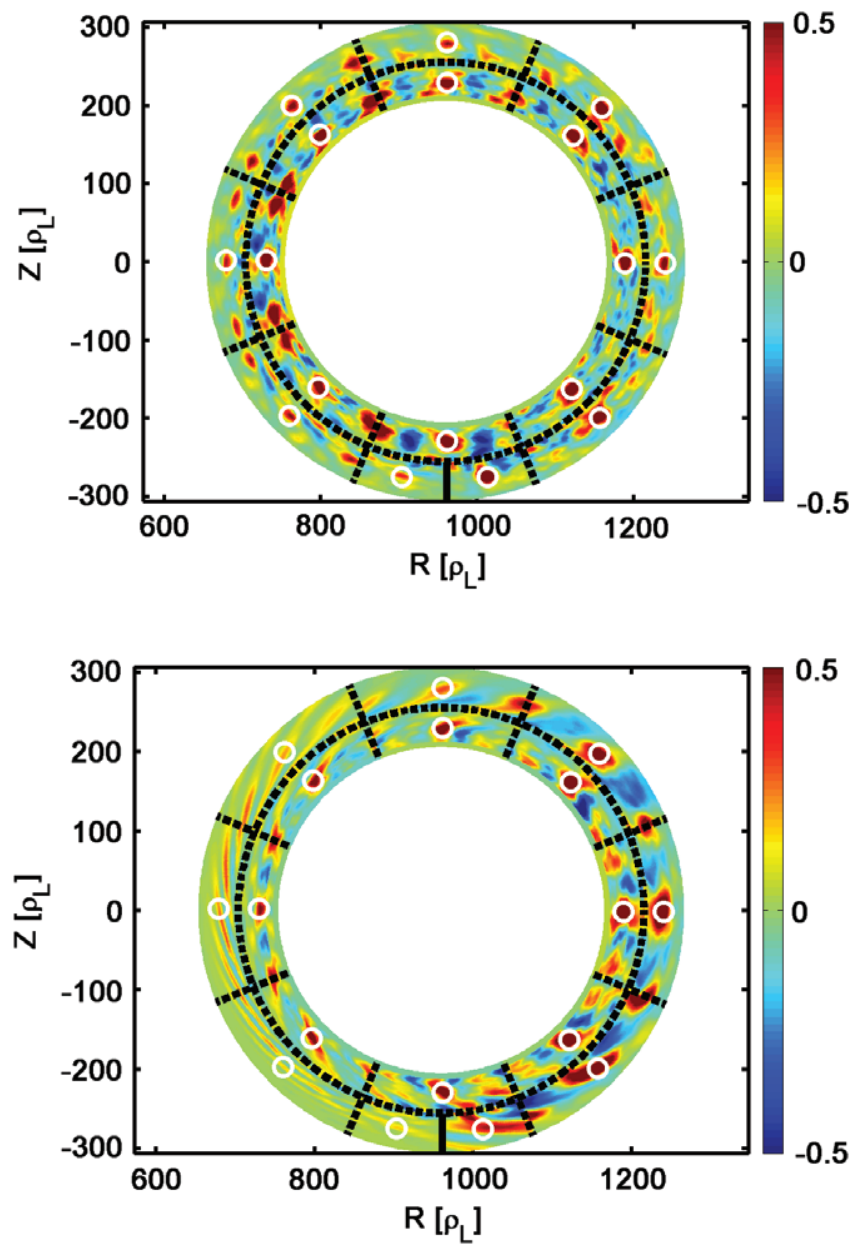

FIGURE 4 Same conditional averaging with parabolic $q^{\text {cyl }}$-profile

FIGURE 5 Density profile at HFS mid-plane for flat and parabolic $q^{\text {cyl }}$-profiles. Vertical red dot lines correspond to resonant magnetic surfaces in the simulation with parabolic profile

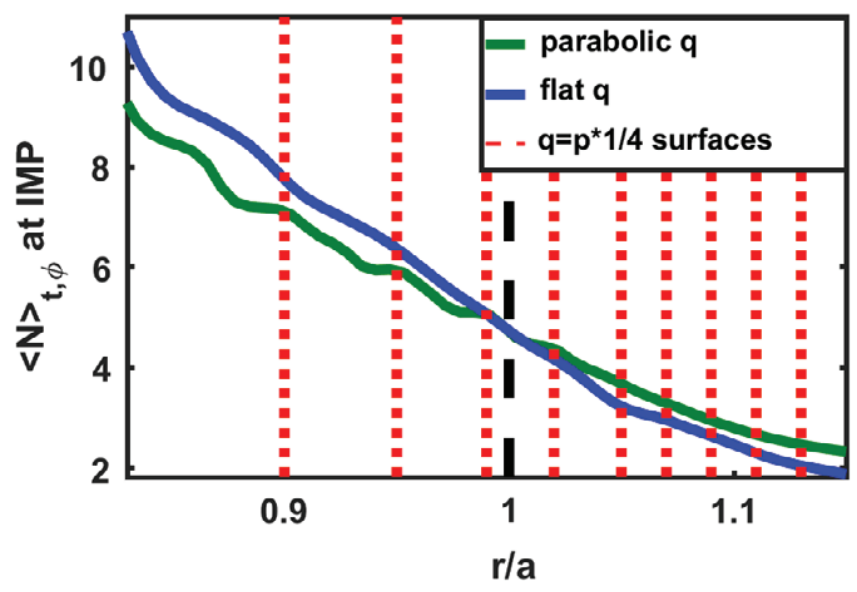

answer this question, HFS and LFS density profiles are plotted, respectively, on Figures 5 and 6 for the two simulations with flat and parabolic $q^{\text {cyl }}$-profiles. The profiles obtained with a flat $q^{\text {cyl }}$-profile are really similar for inboard (IMP) and outboard (OMP) mid-planes such that we can conclude that no strong poloidal dependency and no significant ballooning exist on particle transport without magnetic shear. When the magnetic shear is taken into account, the amplitude of the IMP density profile stays close to the case without magnetic shear, while the profile is strongly flattened at OMP, which is the sign of a strong ballooning. Thus, including magnetic shear has a significant effect on turbulence ballooning and, therefore, on HFS/LFS asymmetry. Finally, a strong correlation is found for the parabolic case between density profile and magnetic surfaces by multiple of quarters of integers. These magnetic surfaces are resonant magnetic surfaces for the code as we only simulate a quart of the torus in the toroidal direction. A flattening of density appears at these resonant magnetic surfaces in closed field lines in HFS, while these resonant surfaces do not act on LFS due to ballooning of turbulence.

The enhancements of ballooning and turbulence by magnetic shear are susceptible to the generation of zonal flows that are able to strongly modify radial transport properties. The generation of zonal flows by turbulence is driven by the Reynolds stress, ${ }^{[5]}$ 

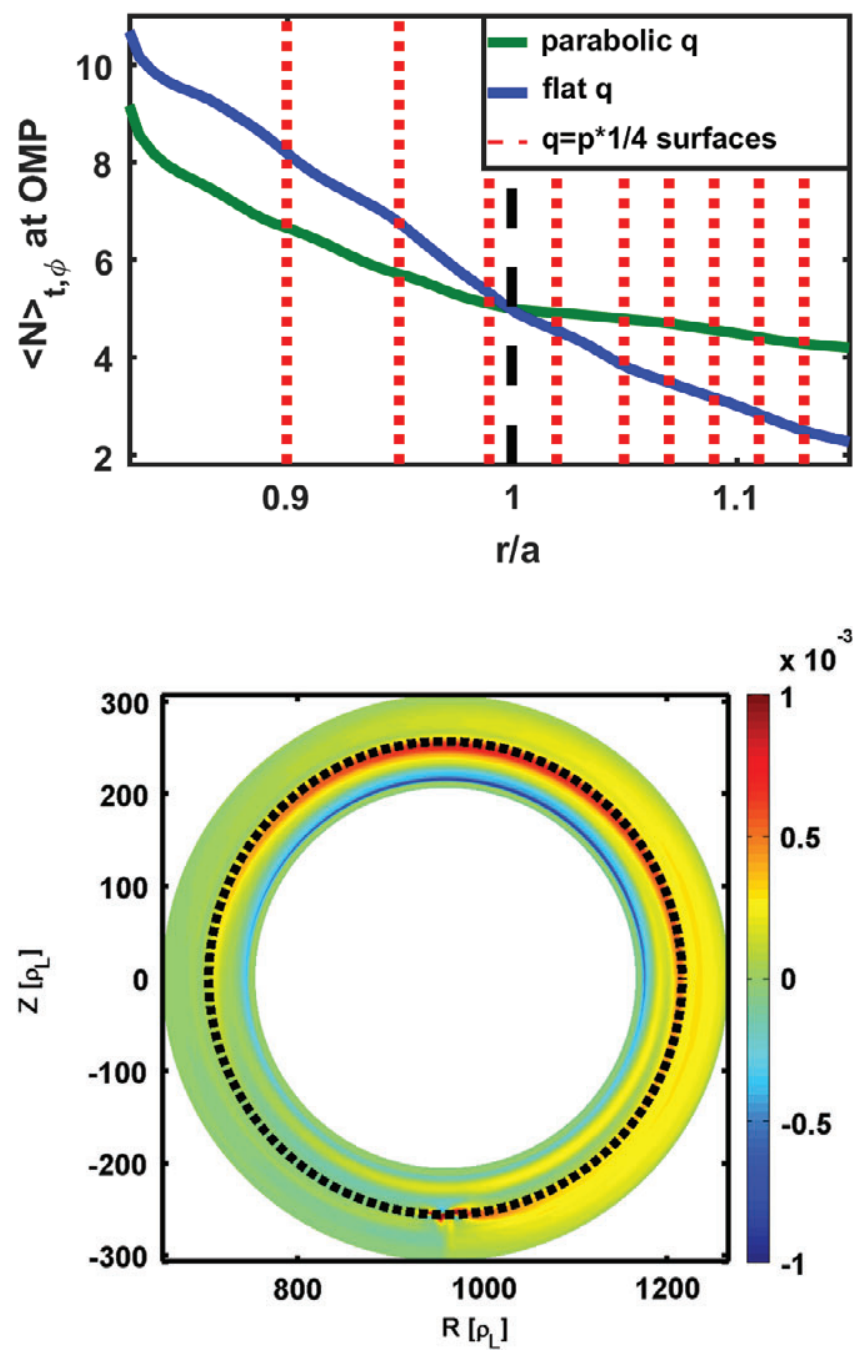

FIGURE 6 Density profile at LFS mid-plane for flat and parabolic $q^{\text {cyl }}$-profiles. Vertical red dot lines correspond to resonant magnetic surfaces in the simulation with parabolic profile

FIGURE 7 Reynolds stress $<\widetilde{u}_{r} \widetilde{u}_{\theta}>_{t, \phi}$ for the simulation with flat $q^{\text {cyl }}$-profile

which plays a key role in transport barrier formation and dynamics. The Reynolds stress (RS) corresponds to the mean product of radial and poloidal velocity fluctuations: $R S=\widetilde{u}_{r} \widetilde{u}_{\theta}$. Poloidal sections of RS are presented in Figures 7 and 8 , respectively, for flat and parabolic $q^{\text {cyl }}$-profile. The same colorbar is applied to both Figures. Without magnetic shear, the RS magnitude does not exceed $5 \times 10^{-4}$, with a maximal magnitude at the top of the device. The RS does not present a significant poloidal asymmetry except close to the LCFS. Conversely, we note a strong enhancement of RS in the presence of magnetic shear with a magnitude larger than $10^{-3}$ for most of the poloidal section. A strong up-down anti-symmetry also appears in the presence of magnetic shear, which may lead to the development of zonal flows. In this section, a strong impact of magnetic shear and safety factor profile on turbulence and transport properties has been established. In the parabolic case, this effect seems, however, too weak to develop zonal flows with amplitudes large enough to damp turbulence and to generate a transport barrier. In the following, we focus on the increase of this magnetic shear in a case that is getting closer to an X-point configuration for which transport barriers are expected to be easier to obtain.

\section{4 | TRANSPORT BARRIER GENERATED BY MAGNETIC SHEAR}

A final simulation-mimicking the flux surface average magnetic shear generated by the presence of an X-point in a COMPASS-like divertor geometry - is performed in our circular limited geometry with safety factor and magnetic shear profiles presented in Figures 1 and 2, respectively. The local effect of the X-point is not included in our simulation as magnetic shear is mostly homogeneous poloidally. The two peaks of magnetic shear on each side of the LCFS have a strong impact on transport properties. Indeed, a strong improvement of particle confinement is obtained in the COMPASS-like $q^{\text {cyl }}$-profile in comparison with flat and parabolic simulations, as can be seen in Figure 9. While the mean density amplitude for the three simulations is nearly the same in open field lines at $r=1.05 a$, the density in the core region is increased by a factor 1.5 and increases from around 9 for the flat and parabolic simulations to 15 for the COMPASS-like magnetic shear simulation. The maxima of density gradient appear in the two regions where the magnetic shear peaks are localized, that is, at $r=0.98 a$ and at $r=1.02 a$. 
FIGURE 8 Reynolds stress $<\widetilde{u}_{r} \widetilde{u}_{\theta}>_{t, \phi}$ for the simulation with parabolic $q^{\mathrm{cyl}}$-profile

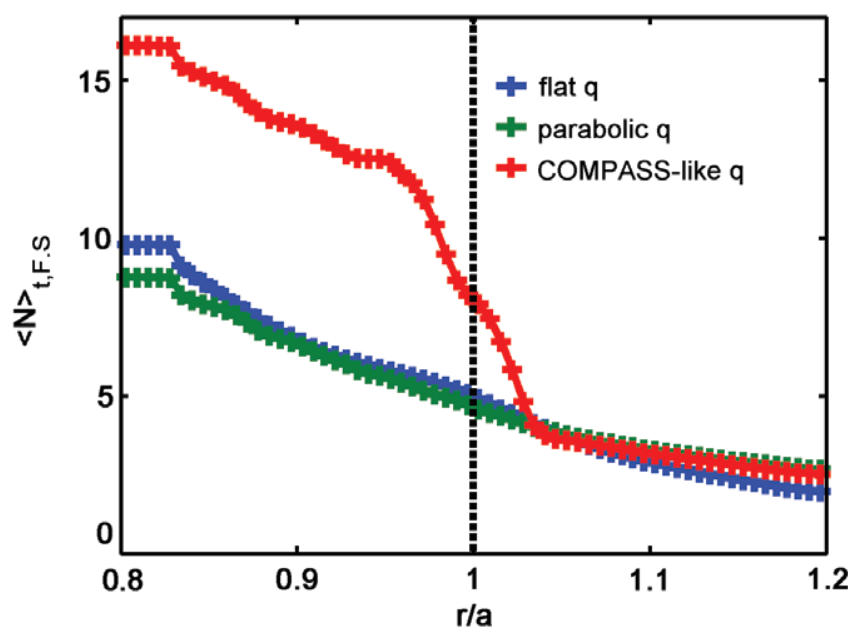

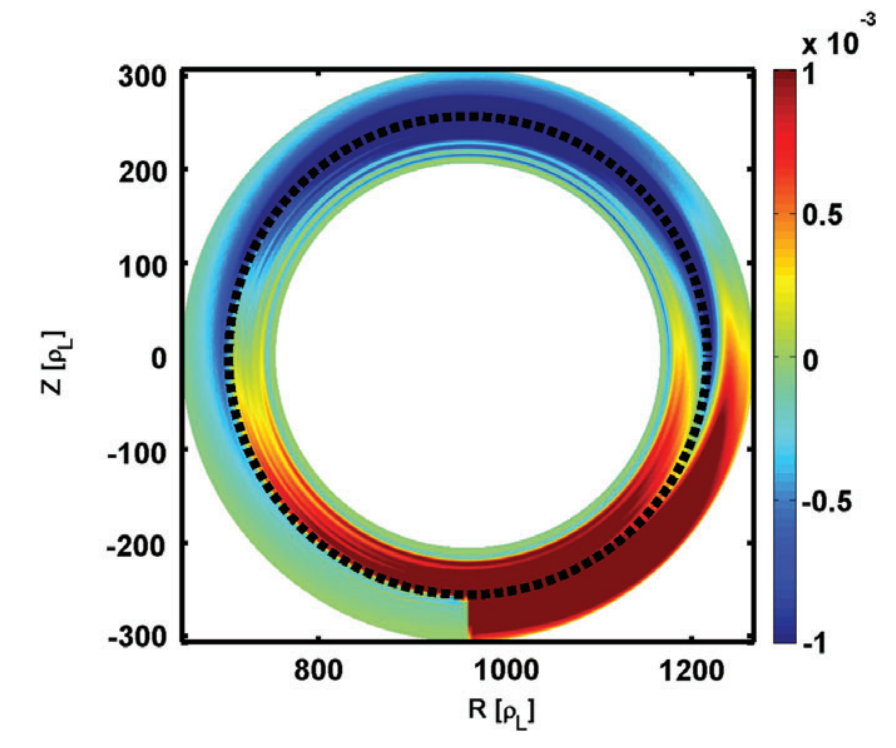

FIGURE 9 Radial profile of flux surface average density for the three simulations with flat, parabolic, or COMPASS-like $q^{\text {cyl }}$-profile. $\langle\cdot\rangle_{F . S}$ corresponds to the flux surface average
In order to achieve a better understanding of how the radial transport is affected by the magnetic shear profile, Figure 10 details the radial profile of flux surface average radial fluxes in the simulation mimicking the X-point configuration. Turbulent $E \times B$ and diffusive and total particle fluxes are displayed in this figure. The total flux is expected to be constant radially in the closed field lines region and decays in the open field line area. With flat and parabolic $q^{\text {cyl }}$-profiles (not plotted here), the total flux and the $E \times B$ cannot be distinguished, and all the transport is ensured by turbulence, the diffusive contribution being negligible. In this simulation mimicking X-point magnetic shear magnitude, one can observe that a significant part of radial transport is coming from diffusive transport, especially on both sides of the LCFS, where the magnetic shear peaks are localized. It is obvious that an increase of density gradient leads to a rise in radial diffusive transport, which represents up to $40 \%$ of radial transport at $r=1.025 a$. The drop of turbulent transport associated with the increase of core particle confinement is a sign of the existence of an edge transport barrier close to the LCFS. One of the main paradigms of edge transport barrier formation is that the turbulence is stabilized by the $E \times B$ velocity shear. ${ }^{[6]}$ Figure 11 presents the radial profile of $E \times B$ shear for the three simulations with varying $q^{\text {cyl }}$-profile. In the simulations without or with a small magnetic shear magnitude-that is, flat and parabolic cases- the $E \times B$ shears have a magnitude lower than $5 \times 10^{-3} \omega_{c}$, with no significant increase at the LCFS. Conversely, a strong rise of $E \times B$ shear magnitude is observed in the COMPASS-like simulation around the LCFS region with two positive peaks on both sides of the LCFS and a large negative one at the LCFS. The enhancement of $E \times B$ shear by magnetic shear is in agreement with calculations from KSTAR measurements. ${ }^{[7]}$ We can wonder which one of the magnetic or $E \times B$ shear is responsible for the formation of the transport barrier. To answer this question, we first introduce a criterion, called the barrier efficiency ${ }^{[8]}$ which measures the capability of a flux surface to stop the radial propagation of turbulence. This criterion—varying between 0 and 1 and depending on time and flux surface index (i.e., radial direction)—corresponds to the proportion of the total radial transport, which is not caused by turbulence: $\varepsilon_{B}(\psi, t)=1-\left\langle\Gamma^{E \times B}\right\rangle_{F . S} /\left\langle\Gamma^{\text {total }}\right\rangle_{F . S}$.

Radial profiles of $E \times B$ shear, magnetic shear, and time-average barrier efficiency are superimposed on Figure 12 for the COMPASS-like simulation and permits the observation of the radial correlation between the two shears and the localization 

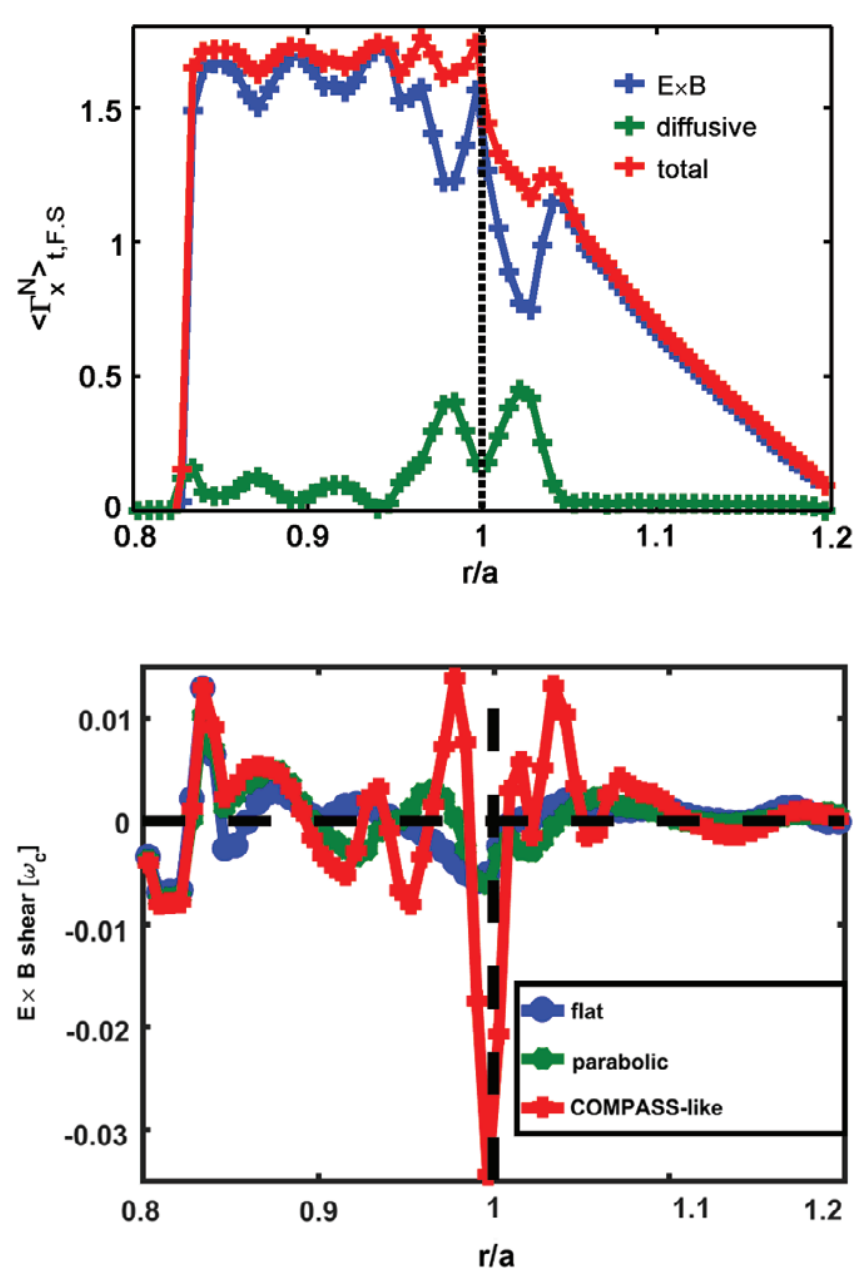

FIGURE 10 Radial profile of flux surface average radial fluxes for the simulation mimicking COMPASS-like divertor $q^{\text {cyl }}$-profile
FIGURE 11 Radial profile of time and flux surface-averaged $E \times B$ shear for the three simulations with flat, parabolic, and X-point like $q^{\text {cyl }}$-profile

of the transport barrier. Concerning the barrier efficiency, one can notice the existence of two transport barriers: the first one is localized in the core region at $r=0.98 a$ with an efficiency of $25 \%$ and the other one in open field lines at $r=1.025 a$ with an efficiency of $37 \%$. The radial position of the two barriers is more correlated with the magnetic shear than the $E \times B$ one. Indeed, the two peaks of magnetic shear coincide quasi-perfectly with the barrier efficiency maxima, while the $E \times B$ shear profile reveal a weaker correlation. In particular, the maximum magnitude of this shear, at the LCFS, does not generate a transport barrier at this radial position. It also seems necessary to presume that the two barriers are not fluctuating in time as it can be observed experimentally, for example, during $\mathrm{L}-\mathrm{H}$ transition. In other words, the stabilization of turbulence is always occurring constantly in this simulation such that the standard predator-prey model cannot be applied in this case. Such absence of interplay between $E \times B$ shear and barrier efficiency, combined with a greater radial correlation with the magnetic shear, demonstrates that the two transport barriers in this simulation result from the existence of a large magnetic shear and not from the enhancement of the $E \times B$ shear by the magnetic one.

\section{5 | CONCLUSION}

In this study, the impact of magnetic configuration on edge turbulence properties in circular limiter geometry has been investigated using a 3D, first-principle, fluid code for edge plasma. Three simulations, one without magnetic shear—one with a small magnetic shear magnitude corresponding to limiter circular geometry, and one mimicking the effect of an X-point-are compared and analysed. First, the expected spatial tilting of turbulence fluctuations is recovered, as well as the anti-symmetry with respect to the LFS mid-plane. We also underline the effect of resonant magnetic surfaces, which flatten density profiles, especially on the HFS. Magnetic shear is found to enhance RS and to generate an up-down anti-symmetry. The modelling of the effect of an X-point in our circular limiter geometry leads to the formation of two transport barriers on both sides of the LCFS. A strong improvement of particle confinement, as well as the reduction of turbulent transport, has been established. A strong increase of $E \times B$ shear is also induced by the magnetic shear. The analysis of spatial correlation between the two shears and of the 
FIGURE 12 Radial profile of $E \times B$ shear, magnetic shear, and mean barrier efficiency for the simulation with COMPASS-like magnetic shear

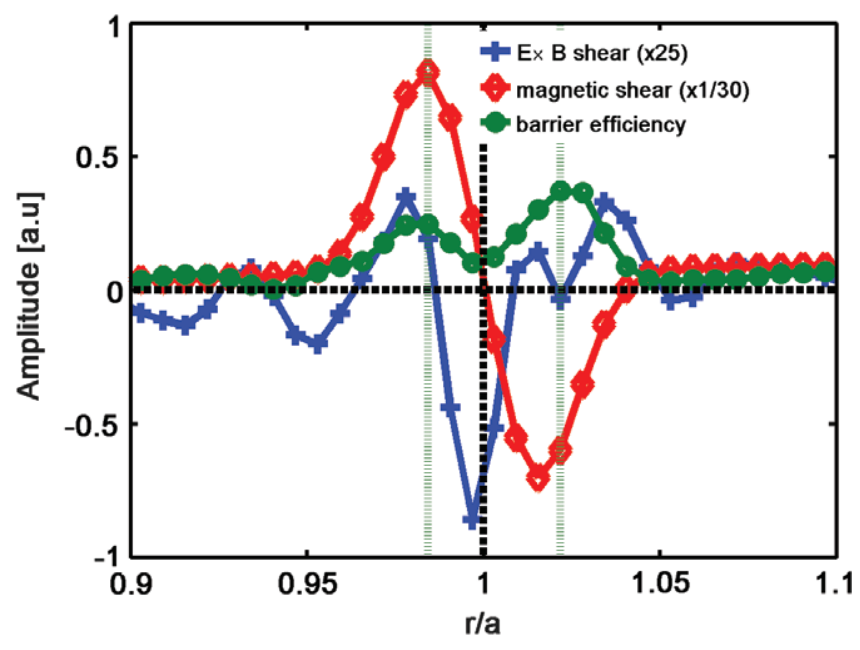

barrier efficiency—which measures the capability of a flux surface to stop the radial propagation by turbulence-demonstrate that the formation of the two transport barriers are induced by the magnetic shear and not the $E \times B$ one.

\section{ACKNOWLEDGMENTS}

This work has been financially supported by the Agence Nationale pour la Recherche (ANR) via project SEDIBA (Blanc SIMI 9 2011). This work has been carried out thanks to the support of the A*MIDEX project (no. ANR-11-IDEX-0001-02) funded by the "Investissements d'Avenir" French Government programme, managed by the French National Research Agency (ANR). It was granted access to the HPC resources of Aix-Marseille Université financed by the project Equip@Meso (ANR-10-EQPX-29-01) of the programme "Investissements d'Avenir" supervised by the ANR. It also used HPC resources from GENCI IDRIS and TGCC in the frame of projects x2016056912 and A0010506912 and from CINECA in the frame of the TOPEDGE project.

\section{REFERENCES}

[1] D. Kalupin, M. Z. Tokar, B. Unterberg, X. Loozen, D. Pilipenko, R. Zagorski and TEXTOR Contributors, Plasma Phys. Control. Fusion 2006, 48 , A309.

[2] N. Fedorczak, P. H. Diamond, G. Tynan, P. Manz, Nucl. Fusion 2012, 52, 10.

[3] P. Tamain, H. Bufferand, G. Ciraolo, C. Colin, D. Galassi, Ph. Ghendrih, F. Schwander, E. Serre, J. Comput. Phys. 2016, 321, 606.

[4] H. Sugama, M. Wakatani, Phys. Fluids 1988, 31, 1601.

[5] K. Itoh, S.-I. Itoh, P. H. Diamond, T. S. Hahm, A. Fujisawa, G. R. Tynan, M. Yagi, Y. Nagashima, Phys. Plasmas 2006, 13, 055502.

[6] K. H. Burrell, Phys. Plasmas 1997, 4, 1499.

[7] T. S. Hahm, D. H. Na, J. W. Lee, J. W. Park, Y. S. Na, S. S. Kim, W. H. Ko, P. H. Diamond, H. Jhang, Y. M. Jeon, Nucl. Fusion 2013, 53, 093005.

[8] N. Nace, M. Perin, P. Tamain, G. Ciraolo, C. Baudoin, R. Futtersack, Ph. Ghendrih, C. Norscini, Contrib. Plasma Physics 2016, 56, 581. 